\title{
Services for Science
}

\author{
Ian Foster \\ Computation Institute \\ Argonne National Laboratory \\ \& University of Chicago \\ foster@mcs.anl.gov
}

\begin{abstract}
Computational approaches to problem solving have proven their worth in many fields of science, allowing the collection and analysis of unprecedented quantities of data and the exploration via simulation of previously obscure phenomena. We now face the challenge of scaling the impact of these approaches from the specialist to entire communities. I speak here about work that seeks to address this goal by rethinking science's information technology foundations in terms of service-oriented architecture. In principle, service-oriented approaches can have a transformative effect on scientific communities, allowing tools formerly accessible only to the specialist to be made available to all, and permitting previously manual data-processing and analysis tasks to be automated. However, while the potential of such "service-oriented science" has been demonstrated, its routine application across many disciplines raises challenging technical problems. One important requirement is to achieve a separation of concerns between discipline-specific content and domainindependent infrastructure, so that new services can be developed quickly and existing services can respond effectively to time-varying load. Another key requirement is to streamline the formation and evolution of the "virtual organizations" that create and access content. I describe the architectural principles, software, and deployments that I am and my colleagues have produced as we tackle these problems, and point to future technical challenges and scientific opportunities. I illustrate my talk with examples from astronomy and biomedicine.
\end{abstract}

